\title{
Active splitting strategy searching approach based on MISOCP with consideration of power island stability
}

\author{
Yifan $\mathrm{ZHOU}^{1}$, Wei HU ${ }^{1}$, Yong MIN ${ }^{1}$, Le ZHENG ${ }^{1,2}$
}

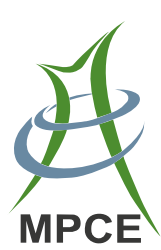

\begin{abstract}
Active splitting control utilizes real-time decision and system-level splitting to prevent cascading blackouts and to maintain power supply under severe disturbances. Splitting strategy searching (SSS) is one of the most crucial issues in active splitting control for deciding "where to split". SSS determines the splitting surface in real time to properly divide the asynchronous generators into isolated islands with an optimal control effect. In this paper, an SSS approach that focuses on island stability is presented. The proposed SSS approach is designed to ensure a rational stability margin and regulation ability on each island during and after the transient process of system splitting. This method includes the active/reactive power flow feasibility constraints and voltage/angle stability constraints in the steady state, as well as the frequency response capability constraints in the transient process. By considering the island stability constraints in the SSS, the proposed approach can avoid the splitting strategies with
\end{abstract}

CrossCheck date: 6 December 2018

Received: 18 July 2018/Accepted: 6 December 2018/Published online: 28 February 2019

(C) The Author(s) 2019

$\bowtie$ Yifan ZHOU

zhou-yf14@mails.tsinghua.edu.cn

Wei HU

huwei@mail.tsinghua.edu.cn

Yong MIN

minyong@mail.tsinghua.edu.cn

Le ZHENG

owenlele@gmail.com

1 Department of Electrical Engineering, Tsinghua University, Beijing 100084, China

2 Department of Cardiothoracic Surgery, Stanford University, Stanford, USA poor stability performance. Therefore, the major advantage of the proposed approach is ensuring better island static and transient stability during and after the splitting control. In addition, the entire model is formulated as a mixedinteger second-order cone programming (MISOCP) model. Thus, it can be rapidly solved by using commercial optimization solvers. Numerical simulation of a realistic provincial power system in central China demonstrates the validity of the proposed approach and the necessity of considering the island stability issues.

Keywords Splitting strategy searching (SSS), Mixedinteger second-order cone programming (MISOCP), Controlled islanding, Static stability margin, Transient stability, Power system

\section{Introduction}

Splitting control is the last defense line in power system security control for preventing system-wide blackouts [1]. When an out-of-step oscillation occurs and conventional emergency control fails to suppress the oscillation, splitting control is activated to separate the asynchronous generator groups into several isolated power islands, thereby restricting fault propagation and maintaining power supply [2].

With the rapid development of power system monitoring and communication in recent years, the concept of active splitting control has been proposed, directing research toward a real-time monitoring, system-level decision and adaptive control approach [3]. In the active splitting control, major tasks include deciding whether to split [4], when to split [5], and where to split [6]. In this paper, we concentrate on the last problem, which is also referred to as the 
splitting strategy searching (SSS) problem. The objective of SSS is to determine a suitable set of transmission lines to be tripped out in real time to separate the asynchronous generator groups. Reference [7] systematically summarized the system operating requirements in SSS as the following four main points: (1) synchronization separation constraint (SSC), i.e., the asynchronous generator groups should be separated into isolated islands; (2) power balance constraint (PBC), i.e., the power generation and load of each island should be balanced; (3) static equilibrium constraint (SEC), i.e., each island can reach a feasible steady-state operating point with a sufficient static stability margin; (4) transient equilibrium constraint (TEC), i.e., each island is capable of stably surviving the transient process.

Numerous studies have been performed to investigate SSC and PBC in SSS, such as ordered binary decision diagram based methods [3, 6], slow coherency methods $[8,9]$, graph theory methods [10, 11], and constrained optimization methods $[12,13]$. It has been shown that SSC and PBC can be accurately modelled in the form of linear constraints and be effectively handled by the existing approaches.

Although SSC and PBC are thoroughly discussed in previous studies, the consideration of SEC and TEC is far from adequate despite their non-negligible importance. The over-simplification of SEC and TEC originally comes from the complexity of the stability constraints. In the majority of SSS studies, SEC is simplified as the power flow feasibility (PFF) constraint on each island. Reference [6] designs a practical multi-phase approach and examines the island PFF of each candidate strategy in the "checking" stage. The research in [14] presents a mixed-integer linear programming (MILP) model to formulate the DC power flow (DCPF) constraints of each island. In addition to PFF, several studies investigate other static stability issues in SSS. Reference [15] proposes a two-stage stochastic approach which considers the possible contingencies on each island to enhance the island reliability. Reference [16] develops the static voltage stability consideration in SSS by checking the Q-V sensitivity of each candidate splitting strategy. Rather than strategy checking, research in [17] investigates the formulation method of island voltage stability margin (VSM) in SSS so that the splitting strategy satisfying certain VSM can be directly solved out. Regarding TEC, the necessity of considering the transient process in the splitting strategy decision has been fully demonstrated by simulations in [18]. However, the precise modelling of TEC in SSS is extremely complex due to the non-linearity introduced by the differential algebraic equations; thus, the majority of previous studies do not consider TEC. Adopting proper island corrective control strategies to assist the splitting control would be an effective way to improve the island transient performance
[19, 20]. However, these approaches do not directly address how to obtain a more rational splitting strategy. Reference [18] proposes a "threshold value constraint" in SSS to restrict the degree of the island disturbance caused by the splitting control. Thus, each power island is more likely to survive the transient process. However, the threshold for the island active power disturbance is empirically determined and fails to take the island regulation ability into account, which may lead to a conservative or aggressive result. Similarly, several studies consider TEC by addressing the island active power disturbance problem caused by the system splitting, such as minimizing the pre-split island power flow exchange [21], minimizing the post-split island power imbalance [22], and so on.

Moreover, SSS with only SSC, PBC and PFF has been proven to be a multiple optimal solution (MOS) problem [23]. This fact indicates that although various splitting strategies exist in which the load loss minimized, these strategies would vary largely in terms of stability performance if the SEC and TEC are lacked.

Because each island is equipped with emergency control, it is unnecessary for the splitting strategy to strictly satisfy every stability constraint. Rather, it would be practical to ensure a rational stability margin and regulation ability on each island. Motivated by this understanding, this paper aims to develop a practical SEC and TEC modelling approach to make a trade-off between the complexity of SEC/TEC models and computational burden. The main contributions of this paper can be summarized as follows. First, the stability risks during the overall splitting control process are discussed. Essential issues that affect the island static and transient stability characteristics are extracted. Second, the modeling approach for the stability issues is investigated. Consequently, the entire SSS model considering island stability appears as a mixed-integer second-order cone programming (MISOCP) problem; thus, it can be rapidly solved using commercial solvers. Third, a numerical simulation is performed in a realistic power system in central China to verify the effectiveness of this study. A comparison between strategies with and without stability considerations is presented to illustrate the necessity of embedding stability issues in SSS.

Compared with the previous studies, this paper addresses the island static and transient stability characteristics both during and after the splitting control. Thus, the splitting strategies with poor stability performance are effectively rejected in the strategy searching process. Additionally, the whole problem is formulated in a deterministic MISOCP form to ensure the problem-solving efficiency and the method practicality. The remainder of this paper is organized as follows. Section 2 briefly analyzes the essential stability issues in SSS as the preliminaries of this research. Section 3 investigates the problem 
formulation in detail. Section 4 provides the method implementation scheme. Section 5 presents the numerical simulation and discussion. Finally, Section 6 draws the conclusions.

\section{Problem analysis}

The overall splitting process can be roughly divided into four periods [7], as presented in Fig. 1. In each period, the stability risks vary due to specific factors and correspond to different SSS constraints. A proper splitting strategy should be capable of managing the major stability risks in each period.

1) Interconnected system operation period

Once system splitting is inevitable following a large disturbance, coherency identification is implemented to distinguish the asynchronous generator groups, and then SSS is activated to determine a splitting control strategy. Generally, the main stability risk during this period comes from an out-of-step oscillation, namely, the transient angle stability problem. This issue corresponds to SSC.

2) System separating period and island restoring period

Once the splitting strategy is determined, the system splitting is activated at a proper time. In the system separating period, the stability risk on each island is primarily affected by the active power imbalance [18]. This issue corresponds to TEC. Directly embedding the detailed transient model in SSS is impractical considering the computational burden. Rather, TEC can be simplified as restricting the impact of the initial power imbalance on each island as recommended by [18].

3) Island operation period

After the island oscillation is damped, each island transitions to a steady-state operating point. For a newly formed power island, it is difficult for the system operators to thoroughly understand its operating characteristics in advance. In addition, since each island is hastily constructed in emergency circumstances, it is likely to be vulnerable. Thus, it is a practical requirement to provide a sufficient static stability margin on each island to enhance its security level. This issue corresponds to PBC and SEC. Compared with PBC, SEC places greater emphasis on reactive power distribution, power flow condition and static stability margin.

As a conclusion, the essential issues impacting the island static and transient equilibrium characteristics can be summarized as four main points. In the steady state, the main problems are the feasibility of the island power flow and the improvement of the island static stability margin. In the transient process, the main problems are the separation of the asynchronous generator groups and the restriction of the power imbalance impact caused by the system splitting action. The following section focuses on the method for modelling the stability issues above, thereby obtaining splitting strategies with better stability performance while ensuring the computational efficiency of the SSS model.

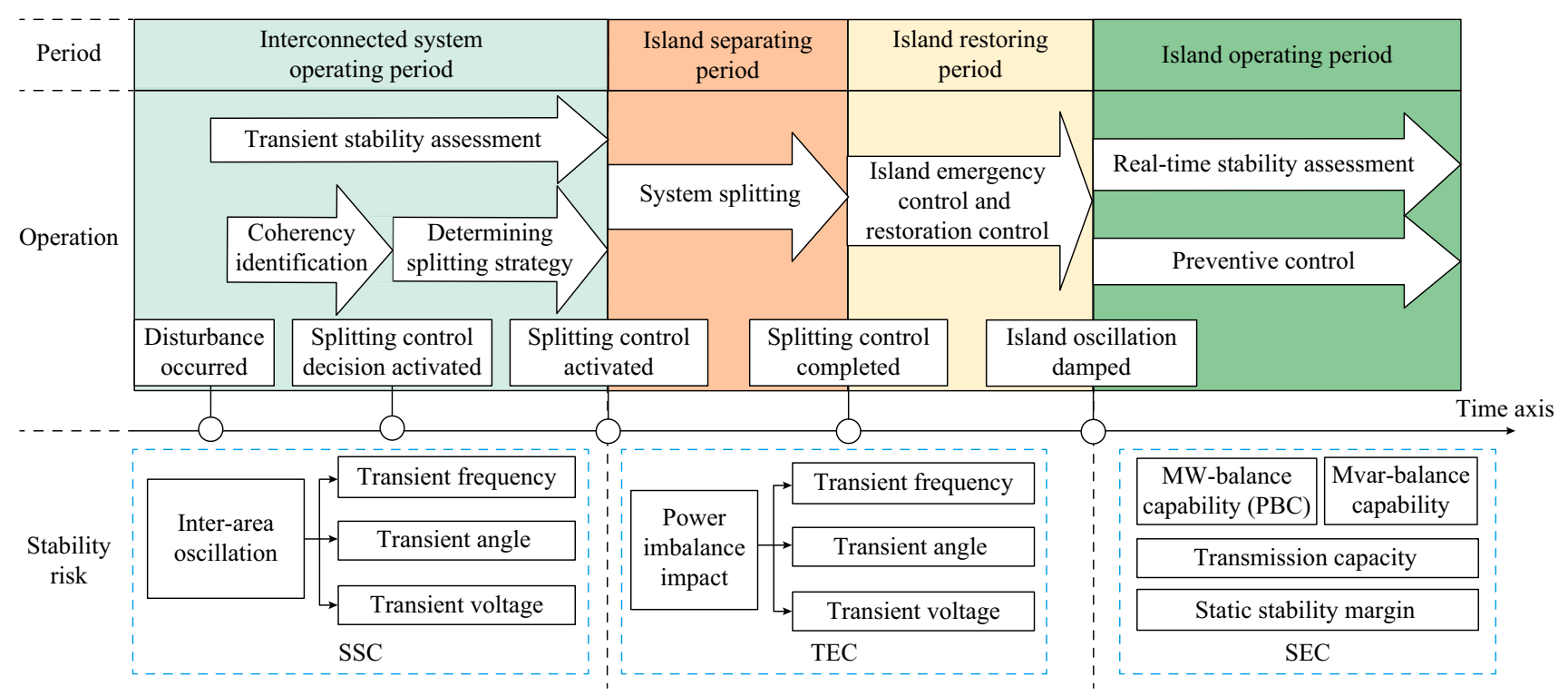

Fig. 1 Illustration of stability risks during the overall splitting control process 


\section{Problem formulation}

This section presents the formulation of the problem with consideration of SEC and TEC in SSS.

\subsection{Formulation for PFF}

The AC power flow model depicts the branch power flow as follows:

$$
\left\{\begin{array}{l}
P_{i j}=V_{i}^{2} g_{i j}-V_{i} V_{j}\left(g_{i j} \cos \theta_{i j}+b_{i j} \sin \theta_{i j}\right) \\
Q_{i j}=V_{i} V_{j}\left(b_{i j} \cos \theta_{i j}-g_{i j} \sin \theta_{i j}\right)-V_{i}^{2}\left(b_{i j}+b_{i j}^{0}\right)
\end{array}\right.
$$

where $P_{i j}, Q_{i j}$ represent the active and reactive power flows from bus $i$ to bus $j ; V_{i}, V_{j}, \theta_{i}, \theta_{j}$ represent the voltage amplitudes and angles at buses $i$ and $j ; \theta_{i j}=\theta_{i}-\theta_{j}$; and $g_{i j}, b_{i j}, b_{i j}^{0}$ denote branch conductance, susceptance and leakage susceptance, respectively.

Since the AC power flow model is non-linear and difficult to solve, an approximate linear power flow model is derived as a substitute for (1). The following proper approximations are adopted: (1) $\sin \theta_{i j} \approx \theta_{i j}$ and $\cos \theta_{i j} \approx 1$ since $\theta_{i j}$ commonly distributes in $[-\pi / 6, \pi / 6]$; (2) the quadratic terms of $\Delta V=V-1$ can be approximately ignored since $V$ commonly distributes in $[0.85,1.1]$.

The above approximations help to establish a linear power flow model. Take the branch active power flow as an example:

$$
\begin{aligned}
P_{i j} & \approx\left(1+\Delta V_{i}\right)^{2} g_{i j}-\left(1+\Delta V_{i}\right)\left(1+\Delta V_{j}\right)\left(g_{i j}+\theta_{i j} b_{i j}\right) \\
& \approx\left(1+2 \Delta V_{i}\right) g_{i j}-\left(1+\Delta V_{i}+\Delta V_{j}\right) g_{i j}-\theta_{i j} b_{i j} \\
& =-b_{i j} \theta_{i}+b_{i j} \theta_{j}+g_{i j} V_{i}-g_{i j} V_{j}
\end{aligned}
$$

Based on (2), the active power flow constraints in SSS can be formulated as:

$$
\begin{aligned}
& \sum_{(i, j) \in E} P_{i j}=P_{i}^{G}-P_{i}^{L} \quad \forall i \in N \\
& P_{i j}=\left(-b_{i j} \theta_{i}+b_{i j} \theta_{j}+g_{i j} V_{i}-g_{i j} V_{j}\right) x_{i j} \quad \forall(i, j) \in E
\end{aligned}
$$

where $N$ is the node set; $E$ is the branch set; $P_{i}^{G}$ and $P_{i}^{L}$ are the active power generation and power load at bus $i$, respectively; and $x_{i j}$ is the binary variable indicating the status of the branch from $i$ to $j$, where $x_{i j}=0$ if the branch is tripped out or 1 otherwise. Constraint (3) ensures the active power balance. Constraint (4) sets the branch active power flow equation.

The multiplication between $x_{i j}$ and $V_{i}, V_{j}, \theta_{i}, \theta_{j}$ introduces non-linearity to (4). However, they can be equivalently reformulated into linear constraints using the reformulation-linearization technique [24]. Take $V_{i} x_{i j}$ as an example. Define $m_{i j}^{V_{i}}=V_{i} x_{i j}$; then, $m_{i j}^{V_{i}}$ has

$$
\left\{\begin{array}{l}
-\Gamma x_{i j} \leq m_{i j}^{V_{i}} \leq \Gamma x_{i j} \\
V_{i}-\Gamma\left(1-x_{i j}\right) \leq m_{i j}^{V_{i}} \leq V_{i}+\Gamma\left(1-x_{i j}\right)
\end{array}\right.
$$

where $\Gamma$ is a sufficiently large positive constant. The linearization of $V_{j} x_{i j}, \theta_{i} x_{i j}, \theta_{j} x_{i j}$ can be performed similarly. Consequently, the active power flow constraints in (3) and (4) are completely linear.

Analogous to (2), (3) and (4), the reactive power flow constraints for SSS can be formulated as:

$$
\begin{gathered}
\sum_{(i, j) \in E} Q_{i j}=Q_{i}^{G}-Q_{i}^{L} \quad \forall i \in N \\
Q_{i j}=\left[-g_{i j} \theta_{i}+g_{i j} \theta_{j}+\left(-b_{i j}-2 b_{i j}^{0}\right) V_{i}\right. \\
\left.+b_{i j} V_{j}+b_{i j}^{0}\right] x_{i j} \quad \forall(i, j) \in E
\end{gathered}
$$

where $Q_{i}^{G}$ and $Q_{i}^{L}$ are variables that represent reactive power generation and power load at bus $i$, respectively.

Therefore, the PFF constraints are formulated in MILP form, as presented in (3)-(7). In addition, the variable bound constraints should be considered. Due to page limitations, these constraints are not presented.

\subsection{Formulation for static stability margin}

Given that the generators in each island are synchronous, the angle stability problem is typically not severe. Thus, the power angle stability margin is formulated by the branch angle difference restriction as recommended in [25]:

$-\pi / 4 \leq\left(\theta_{i}-\theta_{j}\right) x_{i j} \leq \pi / 4 \quad \forall(i, j) \in E$

VSM reflects the ability of maintaining voltage support in the entire system. Multiple indices have been proposed for static VSM assessment. Among these indices, the Lindex [26] is a widely used method. The nodal L-index is defined as follows:

$L_{i}=\left|1-\sum_{j \in N_{G}} F_{i j}^{L G} \dot{V}_{j} / \dot{V}_{i}\right| \quad \forall i \in N_{L}$

where $N_{G}$ is the set of generator buses; $N_{L}$ is the set of load buses (in this paper, the intermediate buses are regarded as a special type of load buses with the power load as zero); $L_{i}$ is the L-index of bus $i$ (for load buses only); $\dot{V}_{i}$ is the voltage of bus $i$ in the complex number field, $\dot{V}_{i}=V_{i} \angle \theta_{i}$; $F_{i j}^{L G}$ is the $i^{\text {th }}$ row, $j^{\text {th }}$ column element of the complex matrix $\boldsymbol{F}^{L G} . \boldsymbol{F}^{L G}$ is obtained from the post-split system admittance matrix $\boldsymbol{Y}$ as follows: 


$$
\left[\begin{array}{c}
\dot{\boldsymbol{I}}^{G} \\
\dot{\boldsymbol{I}}^{L}
\end{array}\right]=\left[\begin{array}{cc}
\boldsymbol{Y}^{G G} & \boldsymbol{Y}^{G L} \\
\boldsymbol{Y}^{L G} & \boldsymbol{Y}^{L L}
\end{array}\right]\left[\begin{array}{c}
\dot{\boldsymbol{V}}^{G} \\
\dot{\boldsymbol{V}}^{L}
\end{array}\right]
$$

where $\dot{\boldsymbol{I}}^{G}, \dot{\boldsymbol{I}}^{L}, \dot{\boldsymbol{V}}^{G}, \dot{\boldsymbol{V}}^{L}$ represent the vectors of complex current injections and voltages at the generator buses and load buses; $\boldsymbol{Y}^{G G}$ represents the submatrix of $\boldsymbol{Y}$ by extracting the rows and columns corresponding to the generator buses; $\boldsymbol{Y}^{G L}$ represents the submatrix of $\boldsymbol{Y}$ by extracting the rows corresponding to the generator buses and the columns corresponding to the load buses; $\boldsymbol{Y}^{L G}$ and $\boldsymbol{Y}^{L L}$ are defined in analogy. In matrix form, $\boldsymbol{F}^{L G}=$ $-\left(\boldsymbol{Y}^{L L}\right)^{-1} \boldsymbol{Y}^{L G}$ [26]. Furthermore, in element form, $F_{i j}^{L G}$ satisfies the following equation:

$Y_{i j}^{L G}=-\sum_{k \in N_{L}} Y_{i k}^{L L} F_{k j}^{L G} \quad \forall i \in N_{L}, \forall j \in N_{G}$

where $Y_{i k}^{L L}$ and $Y_{k j}^{L G}$ are elements of $\boldsymbol{Y}^{L L}$ and $\boldsymbol{Y}^{L G}$.

A smaller $L_{i}$ indicates a better VSM, and the system VSM is assessed by the maximum nodal VSM [26]. To eliminate the complex number field calculation in (9), the complex-form L-index is defined as follows:

$\dot{L}_{i}=1-\sum_{j \in N_{G}} F_{i j}^{L G} \dot{V}_{j} / \dot{V}_{i} \quad \forall i \in N_{L}$

Equations (11) and (12) help yield the following:

$$
\begin{aligned}
\sum_{k \in N_{L}} Y_{i k}^{L L}\left(\dot{V}_{k} \dot{L}_{k}\right) & \\
& =\sum_{k \in N_{L}} Y_{i k}^{L L} \dot{V}_{k}-\sum_{j \in N_{G}} \dot{V}_{j}\left(\sum_{k \in N_{L}} Y_{i k}^{L L} F_{k j}^{L G}\right) \\
& =\sum_{k \in N_{L}} Y_{i k}^{L L} \dot{V}_{k}+\sum_{j \in N_{G}} Y_{i j}^{L G} \dot{V}_{j}=\dot{I}_{i} \quad \forall i \in N_{L}
\end{aligned}
$$

where $\dot{I}_{i}$ is the complex current injection at bus $i$.

Therefore, the power injection at load buses can be expressed by the L-indices rather than the current injection:

$\dot{S}_{i}=\dot{V}_{i} \dot{I}_{i}^{*}=\dot{V}_{i}\left(\sum_{j \in N_{L}} Y_{i j}^{L L} \dot{V}_{j} \dot{L}_{j}\right)^{*}=\sum_{j \in N_{L}}\left(Y_{i j}^{L L}\right)^{*} \dot{V}_{i} \dot{V}_{j}^{*} \dot{L}_{j}^{*}$

where $\dot{S}_{i}$ represents the complex power injection at bus $i$; * is the conjugate symbol.

Denote $L_{\mathrm{r}, i}$ and $L_{\mathrm{i}, i}$ as the real part and imaginary part of $\dot{L}_{i}$. The following proper approximations hold:

1) For each branch, $g_{i j} \ll b_{i j}$; thus, $Y_{i j} \approx-\mathrm{j} b_{i j} x_{i j} \triangleq \mathrm{j} b_{i j}^{\prime}$, $Y_{i i} \approx \sum_{(i, j) \in E} \mathrm{j}\left(b_{i j}+b_{i j}^{0}\right) x_{i j} \triangleq \mathrm{j} b_{i i}^{\prime}$, where $\mathrm{j}$ is the imaginary unit.

2) For each branch, $V_{i} \approx V_{j}$.

3) For each node $i, V_{i} \approx 1$; thus, $V_{i}^{-2} \approx-2 V_{i}+3$.
Based on the above approximations, (14) can be derived into the following:

$$
\begin{aligned}
\dot{S}_{i} & \approx \sum_{j \in N_{L}}\left(\mathrm{j} b_{i j}^{\prime}\right)^{*} V_{i}^{2} \dot{L}_{j}^{*}=\sum_{j \in N_{L}}-\mathrm{j} b_{i j}^{\prime} V_{i}^{2}\left(L_{\mathrm{r}, j}-\mathrm{j} L_{\mathrm{i}, j}\right) \\
& =\left(-V_{i}^{2} \sum_{j \in N_{L}} b_{i j}^{\prime} L_{\mathrm{i}, j}\right)-\mathrm{j}\left(V_{i}^{2} \sum_{j \in N_{L}} b_{i j}^{\prime} L_{\mathrm{r}, j}\right) \quad \forall i \in N_{L}
\end{aligned}
$$

By separating the real and imaginary parts, (15) is converted into the real number field with proper approximations:

$$
\left\{\begin{array}{l}
\sum_{j \in N_{L}} b_{i j}^{\prime} L_{\mathrm{i}, j}=V_{i}^{-2} P_{i}^{L} \approx P_{i}^{L}\left(-2 V_{i}+3\right) \\
\sum_{j \in N_{L}} b_{i j}^{\prime} L_{\mathrm{r}, j}=V_{i}^{-2} Q_{i}^{L} \approx Q_{i}^{L}\left(-2 V_{i}+3\right)
\end{array}\right.
$$

where $\forall i \in N_{L}$. The multiplication between $b_{i j}^{\prime}$ and $L_{\mathrm{i}, j}, L_{\mathrm{r}, j}$ can readily be converted into the equivalent linear form using the linearization technique presented in (5). Meanwhile, since the priority for splitting control is to minimize load loss to the greatest extent [8], $P_{i}$ and $Q_{i}$ are approximately replaced by the pre-split nodal power load, denoted as $P_{i}^{L 0}$ and $Q_{i}^{L 0}$, respectively.

Therefore, the island VSM assessment model based on the L-index is finally formulated as the following:

$$
\left\{\begin{array}{lll}
\min & O_{S E C}=L_{s y s} & \\
\text { s.t. } & \sum_{j \in N_{L}} b_{i j}^{\prime} L_{\mathrm{i}, j}=P_{i}^{L 0}\left(-2 V_{i}+3\right) & \forall i \in N_{L} \\
& \sum_{j \in N_{L}} b_{i j}^{\prime} L_{\mathrm{r}, j}=Q_{i}^{L 0}\left(-2 V_{i}+3\right) & \forall i \in N_{L} \\
& L_{\mathrm{r}, i}^{2}+L_{\mathrm{i}, i}^{2} \leq L_{s y s} & \forall i \in N_{L}
\end{array}\right.
$$

The first and second constraints in (17) separately calculate the real and imaginary parts of the nodal Lindices. The third constraint in (17) defines the system Lindex $L_{s y s}$ in the second-order cone form. Then, by taking $L_{s y s}$ as the minimization objective $O_{S E C}$, the VSM of each island can be better guaranteed in the SSS decision. Consequently, the island VSM requirement is modeled as a MISOCP model.

\subsection{Formulation of frequency dip/rise restriction}

Sections 3.1 and 3.2 deal with the steady-state stability performance on each island. Further, this subsection discusses the transient process consideration in splitting control. 
Actually, to completely capture the transient instability process is extremely complicated and unwieldy, which is also hard to meet the real-time decision requirement of splitting control. Thus, it is necessary to make a trade-off between the requirement of handling the stability issues and the requirement of real-time decision. It is commonly agreed that the sudden active power imbalance is the primary and the severest threat to the island transient stability after system splitting [18, 20-22, 27]. A large power imbalance disturbance caused by the splitting action would possibly lead to insufferable frequency dip/rise on the islands, and severely threaten the island transient process. Thus, the transient frequency process and the frequency response ability of each island would be a major impact factor for ensuring island transient stability [18]. As for the transient angle stability, since the asynchronous generators are divided into different islands, the generators within each island are basically coherent which largely ensures the island angle stability. Meanwhile, since the reactive power is balanced locally, the transient voltage stability is neither a major issue for most scenarios if the system possesses adequate reactive power reserves and strong enough excitation systems. Consequently, the transient angle and voltage stability problems on each island are usually not severe if a proper splitting strategy is activated timely to separate the generators into isolated islands.

As a result, this subsection focuses on the frequency variation during the island transient process after the sudden power imbalance caused by the splitting action. In the following, a simplified primary frequency response model is introduced to evaluate the power imbalance endurance capability of each island.

Since the generators within each island are coherent, the transient frequency process of each island can be described by the equivalent single-machine model. Accordingly, the swing equation of the equivalent single-machine model for island $d$ can be depicted as follows [28]:

$P_{d}^{i m b}(t)=P_{d}^{m e c}(t)-P_{d}^{\text {elec }}(t)=2 H_{d} \frac{\mathrm{d} \Delta f_{d}(t)}{\mathrm{d} t}$

where $P_{d}^{\text {mec }}(t), P_{d}^{\text {elec }}(t)$ represent the mechanical and electrical power of island $d$ at time $t ; P_{d}^{i m b}(t)$ denotes the mechanical-electrical power imbalance; $H_{d}$ is the island generator inertia; and $f_{d}(t)$ is the island frequency at time $t$.

Additionally, several approximations introduced in (18) should be clarified:

1) The time scale of the transient frequency variation immediately after splitting control mainly corresponds to the time scale of primary frequency response (5-30 s [28]). Thus, the regulation by automatic generation control is roughly ignored.
2) The power loads are modelled as the PQ buses and the change of power load over frequency and voltage is not considered.

3) The damping effect of the power loads and the generators is roughly ignored. This approximation leads to a conservative estimate of the island frequency response ability, since the damping effect would improve the frequency deterioration [29, 30].

4) Although the island frequency differs across different buses, in this paper the average frequency defined by the center of inertia method [28] is adopted.

Suppose that the system is split at time $t=t_{0}$. With a sudden power imbalance $P_{d}^{i m b}\left(t_{0+}\right)$, the island frequency starts suffering from a dip or rise. The initial island power imbalance is expressed as follows:

$P_{d}^{i m b}\left(t_{0+}\right)=P_{d}^{m e c}\left(t_{0+}\right)-\left(P_{d}^{e l e c}\left(t_{0+}\right)-\Delta P_{d}^{i s l}\right)$

where $\Delta P_{d}^{i s l}$ is the load shedding amount on island $d$ after system splitting restricted by the nadir-based frequency stability requirement. Since the generators on each island are pre-decided by coherency identification, $P_{d}^{i m b}\left(t_{0+}\right)$ is impacted by the division of the load nodes.

Responding to the island frequency variation, the generator governors automatically regulate the mechanical power to re-establish the mechanical-electrical power balance. An inadequate frequency-response reserve capability would potentially induce a clear or even insufferable frequency dip/rise. This paper discusses the situation where $P_{d}^{i m b}\left(t_{0+}\right)<0$, corresponding to potential load shedding, as an example. The situation for $P_{d}^{i m b}\left(t_{0+}\right)>0$ can be modeled by analogy. A conservative assumption of a constant generator ramping rate is adopted [29] for simplification. Thus, the island power imbalance is expressed as follows:

$P_{d}^{i m b}(t)=P_{d}^{i m b}\left(t_{0+}\right)+\left(t-t_{0}\right) R_{d} \quad t \geq t_{0}$

where $R_{d}$ is the total governor ramping rate on island $d$. In contrast to the steady-state load shedding, this is a "temporary load shedding" caused by the frequency dynamics and can be recovered after the transient process.

Denote $t_{\text {nadir }}$ as the time that the frequency trajectory meets the first nadir. Figure 2 illustrates the governor regulation process and the corresponding frequency variation. It is easy to understand that $P_{d}^{i m b}\left(t_{\text {nadir }}\right)=0$.

With (18) integrated from $t=t_{0}$ to $t=t_{\text {nadir }}$ and (20) substituted, the expression for the frequency dip can be derived as follows: 


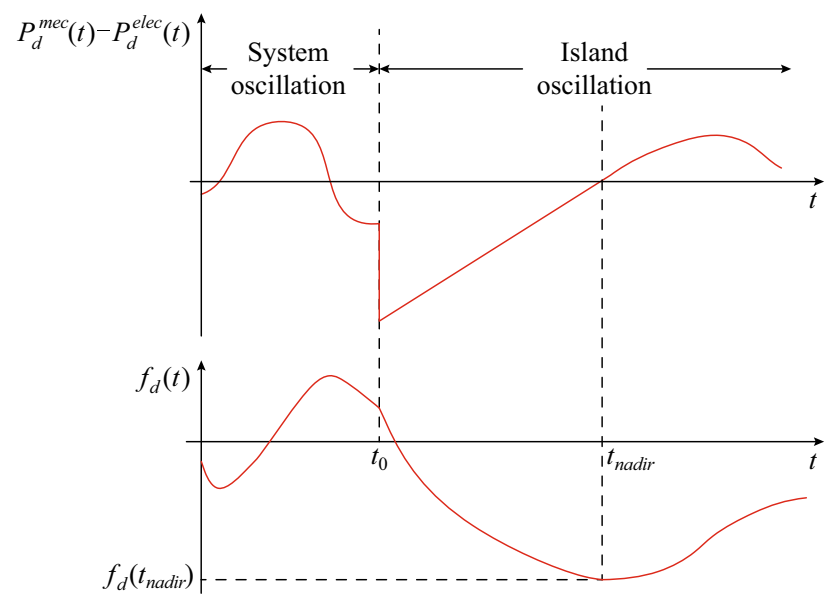

Fig. 2 Island frequency response illustration

$$
\begin{aligned}
\Delta f_{d} & =f_{d}\left(t_{0}\right)-f_{d}\left(t_{\text {nadir }}\right)=-\int_{t_{0}}^{t_{\text {nadir }}} \frac{P_{d}^{i m b}(t)}{2 H_{d}} \mathrm{~d} t \\
& =-\int_{t_{0}}^{t_{\text {nadir }}} \frac{\left(t-t_{0}\right) R_{d}+P_{d}^{i m b}\left(t_{0+}\right)}{2 H_{d}} \mathrm{~d} t \\
& =\frac{\left(P_{d}^{i m b}\left(t_{0+}\right)\right)^{2}}{4 H_{d} R_{d}}
\end{aligned}
$$

To restrict the frequency nadir to a rational range, the frequency dip is restricted as $\Delta f_{d} \leq \Delta f_{\max }$. Therefore, based on (21), the minimal island temporary load shedding constraint is formulated:

$$
\begin{aligned}
\Delta P_{d}^{\text {isl }} \geq & \max \left\{P_{d}^{\text {elec }}\left(t_{0+}\right)-P_{d}^{\text {mec }}\left(t_{0+}\right)\right. \\
& \left.-\sqrt{4 H_{d} R_{d} \Delta f_{\max }}, 0\right\}
\end{aligned}
$$

Constraint (22) depicts the inevitable load loss considering the island inertia and the governor regulation capability during the frequency dynamics. As illustrated in (22), the rational division of the load nodes will greatly impact the frequency dynamics, which also indicates the necessity of considering the frequency response capability. Since SSS is generally controlled in a sufficiently short time, the generator mechanical power at the decision-start time is used to approximate $P_{d}^{\text {mec }}\left(t_{0+}\right)$ with a reasonable assumption that the mechanical power of each generator does not considerably change during the SSS process. Hence, $P_{d}^{\text {mec }}\left(t_{0_{+}}\right)$is known parameters for each island. The equivalent island inertia constant $H_{d}$ and the equivalent island ramping rate $R_{d}$ depend on the generator parameters and the division of generators on each island. Since coherency identification distinguishes the coherent generator groups and pre-determines the generator division before SSS, $H_{d}$ and $R_{d}$ can be pre-calculated based on the generator inertia and governor ramping rate as well as the coherency identification results. Hence, $H_{d}$ and
$R_{d}$ are known parameters for each island independent to the splitting strategy. As a result, the only decision variables are $\Delta P_{d}^{\text {isl }}$ and $P_{d}^{\text {elec }}\left(t_{0+}\right)$, which makes (22) a linear constraint.

Compared with the transient disturbance restriction in [18], the power imbalance restriction in (22) reflects the island frequency response capability rather than an empirical threshold. Meanwhile, because the transient frequency process is modeled as linear constraints, it does not introduce considerable computational burden for SSS.

\subsection{Integrated SSS model}

Based on the formulation work presented above, the SSS model considering the static and transient stability constraints is finally integrated as a MISOCP model:

$$
\begin{cases}\min & O=\lambda_{1} O_{\text {basic }}+\lambda_{2} O_{\mathrm{SEC}}+\lambda_{3} O_{\mathrm{TEC}} \\ \text { s.t. } & O_{\text {basic }}=\sum_{i \in N_{L}}\left(P_{i}^{L 0}-P_{i}^{L}\right) \\ & O_{\mathrm{SEC}}=L_{s y s} \\ & O_{\mathrm{TEC}}=\sum_{d=1}^{D} \Delta P_{d}^{i s l} \\ & (3),(4),(6),(7),(8),(17),(22)\end{cases}
$$

Denote the proposed SSS model (23) as M0. M0 is a threeobjective optimization model. $O_{\text {basic }}$ is the steady-state load shedding, which is the commonly adopted objective in SSS. $O_{\mathrm{SEC}}$ and $O_{\mathrm{TEC}}$ respectively refer to the SEC objective ((17)) and the TEC objective $((22))$. In this paper, the weighted method is simply adopted to address the multiobjective problem. The weight coefficients are set as $\lambda_{1}=100 / \sum_{i \in N_{L}} P_{i}^{L 0}, \lambda_{2}=1$, and $\lambda_{3}=20 / \sum_{i \in N_{L}} P_{i}^{L 0}$ to make minimizing load shedding the top priority. The constraints in (23) include the PFF, island stability margin and transient frequency restriction. Moreover, SSC should also be considered in M0, which is a set of linear constraints. Due to page limitations, please refer to (15)-(18) in [17] for details.

Based on M0, the active splitting strategy is obtained with an appropriate consideration of island stability characteristics. Furthermore, a brief analysis of the feasibility, solvability and adaptability of M0 is provided below. These features are necessary for guaranteeing the practicability of the proposed method.

\subsubsection{Model feasibility}

Real-time control requires the SSS model to be definitely feasible. Otherwise, the system operator will fail to 
obtain the splitting strategy in time, which may lead to disturbance propagation or even a system-wide blackout. Although several complicated stability issues are embedded in M0, it can be proven that the newly considered constraints do not affect the model feasibility. Figure 3 illustrates the feasible region (FR) of the decision variables $\left\{x_{i j} \mid \forall(i, j) \in E\right\}$. With different model constraints, the FR of $x_{i j}$ does not change, which guarantees the model feasibility. Specifically, the power flow constraints in (3)-(7) can always be satisfied by rational generator tripping or load shedding; thus, PFF does not affect the FR for $x_{i j}$, as shown in (2) of Fig. 3. The VSM consideration in (17) is actually an equivalent presentation for solving the L-index, so it does not shrink the FR, as shown in (3) of Fig. 3. The TEC in (22) represents the lower limit of temporary load shedding and does not lead to a break in feasibility. Consequently, the model (23) always has a feasible solution. Additionally, because the optimal solution region (OSR) shrinks when considering more stability constraints, the strategies with poor stability performance are naturally excluded. Therefore, the computational time for further checking the PFF and island stability can be effectively saved [18].

\subsubsection{Model solvability}

Considering the real-time decision requirement of splitting control, the SSS model is supposed to be solved in a short time. Any convergence problems that occur during optimization would be a risk of real-time splitting decision failure. Although the VSM constraint (17) introduces nonlinearity, it is a special type of non-linear constraint, i.e., the second-order cone constraint. Second-order cone programming (SOCP) has an essence of convex programming,

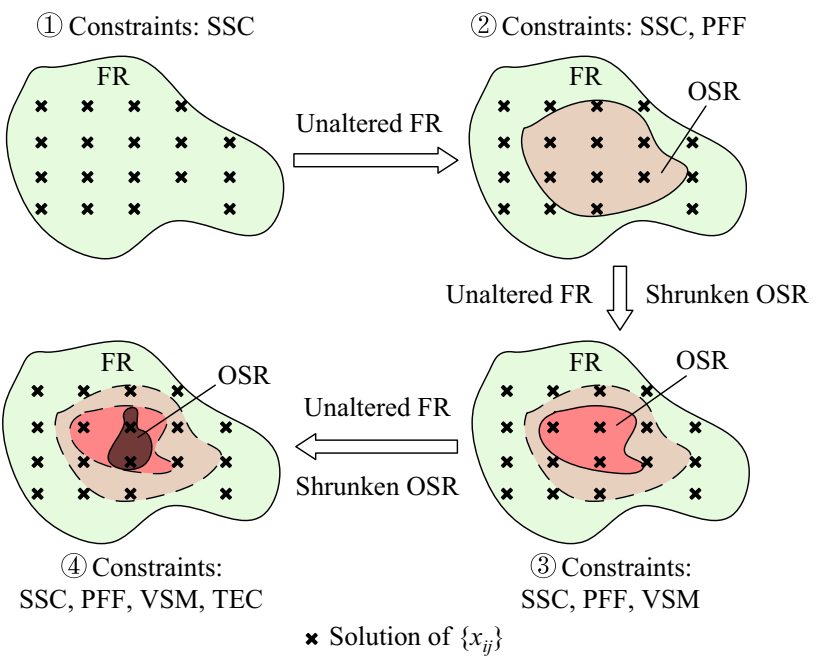

Fig. 3 Illustration of FR and OSR defined in space of $\left\{x_{i j} \mid \forall(i, j) \in E\right\}$ and it exhibits excellent features in optimality and problem-solving efficiency. Mathematically, SOCPs can converge to the global-best solution in polynomial time using interior-point methods [31]. Furthermore, MISOCP can be conveniently and effectively solved by mature algorithms, combining the techniques in integer programming and SOCP, as well as by commercial optimization software, i.e., CPLEX. Consequently, the optimality, convergence and problem-solving efficiency features of (23) are guaranteed, which makes the proposed model solvable in practice.

\subsubsection{Model adaptability}

In addition to feasibility and solvability, it is also important for the SSS approach to work well when the inter-area oscillation between multiple generator groups occurs. Here, we demonstrate that each stability issue considered in the proposed SSS model (23), namely PFF, VSM and TEC, is adaptive to the multiple-group oscillation case. Specifically, for the PFF formulation, the power flow within each island is guaranteed by (4) and (7) with $x_{i j}=1$, while the power exchange between different islands is restricted as zero with $x_{i j}=0$. This formulation holds for arbitrary island number. For the VSM formulation, the voltage support between different islands is eliminated by the zero elements in $\boldsymbol{F}^{L G}$ in (9). Hence the VSM assessment based on L-index remains valid on each post-split island. For the TEC formulation, (22) calculates the load shedding amount restricted by the frequency of each island, which is also valid irrespective of the number of islands. Consequently, model (23) has a good adaptability for separating multiple generator groups. The method performance for the multiple-group oscillation case is also illustrated by the case study in Section 5 .

\section{Implementation scheme}

To illustrate how the active splitting control should be implemented with the proposed method performing SSS, and what monitoring information is required in this whole process, this section discusses the implementation scheme for power system splitting. The structure for active splitting control is presented in Fig. 4. The splitting control scheme consists of two main modules, i.e., the stability assessment module and the splitting execution module.

The first module performs disturbance detection, transient stability assessment and oscillation analysis with the assist of real-time monitoring from the wide-area monitoring system (WAMS) and the supervisory control and 


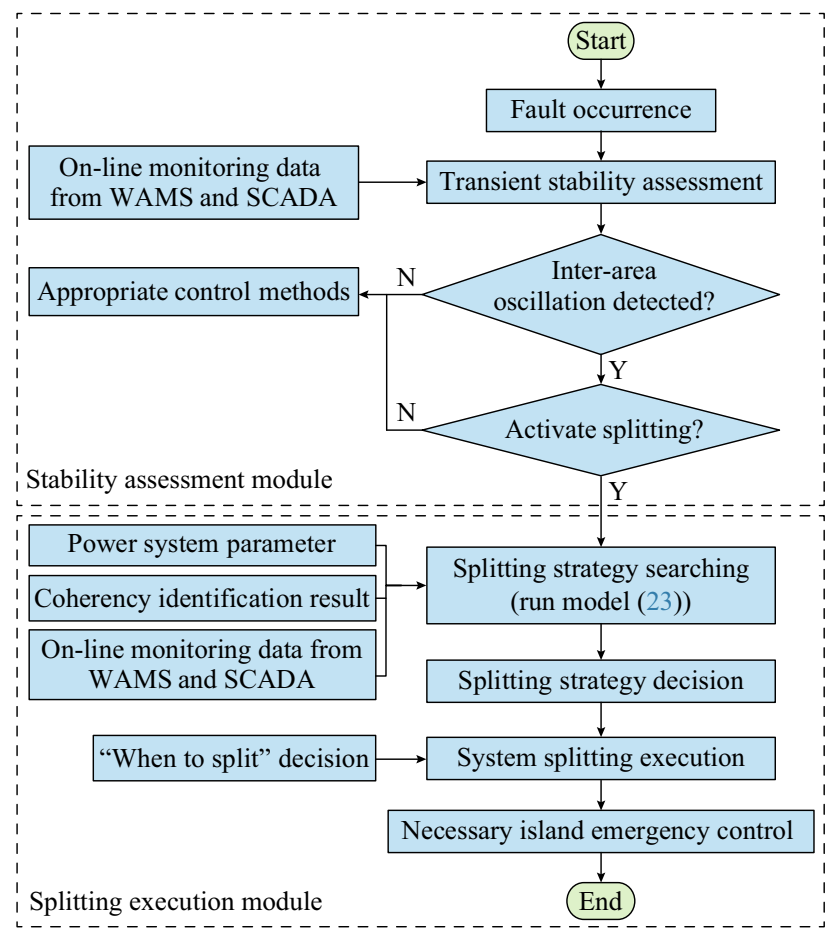

Fig. 4 Structure of active splitting control scheme

data acquisition (SCADA) systems [4]. For this part, necessary real-time information includes the real-time bus angle and voltage, generator rotor angle, system frequency, etc. Since transient stability assessment is not the main concern of this paper, we do not discuss much about this module.

Once the necessity of splitting control is affirmed by the first module, the second module carries out the decision and execution work for splitting control, in which deciding "where to split" is a core section and is done by the proposed SSS approach. More specifically, the coherency identification is firstly performed as a preliminary work to identify the asynchronous generator groups based on the real-time generator rotor angle data [32, 33]. Therefore, the number of the islands in SSS is determined by the number of the asynchronous generator groups, and the division of the generators is determined by the coherency identification result. Then, based on necessary power system parameters (i.e., power grid topology and branch parameters $g_{i j}, b_{i j}, b_{i j}^{0}$ in (3)-(7) and (17), generator parameters $H_{d}, R_{d}$ in (22)), on-line monitoring data (i.e., power load condition before fault occurrence $P_{i}^{L 0}, Q_{i}^{L 0}$ in (17)), and real-time monitoring data (i.e., generator mechanical power $P_{d}^{m e c}$ in (22)), the SSS process is activated by running the MISOCP model presented in (23). Nextly, together with the decision of a proper splitting time [5, 34], the splitting control is executed to perform system separation. Further, after the system splitting, necessary emergency control strategies such as under frequency load shedding, over frequency generator tripping, etc. would be separately adopted on each island for oscillation damping.

\section{Numerical simulation}

This section presents the numerical simulation in a realistic provincial power system in central China, referred to as RPPS. All algorithms are coded in MATLAB R2016a. The commercial optimization software CPLEX12.6 is utilized as the MISOCP solver. Coherency identification is performed using the principal component analysis method [32]. A splitting strategy space reduction method [17] is employed to enhance the optimization efficiency.

\subsection{System introduction and fault condition}

RPPS is abstracted from the $220 \mathrm{kV} / 500 \mathrm{kV}$ network of a realistic power system. As shown in Fig. 5, RPPS is composed of 40 generators, 250 buses and 369 branches. It is a typical hydro-thermal power system, with hydro power mainly in the southwestern area and thermal power mainly in the central and eastern areas. This feature makes the potential power islands differ in terms of regulation and reserve capacity.

Two typical faults inducing the inter-area oscillation are considered, as shown in Fig. 5. In the following, the validity and necessity of the proposed SSS approach is investigated based on these faults.

\subsection{Method validity analysis}

\subsubsection{A two-group oscillation case}

Set fault 1 as a three-phase short circuit fault lasting for $0.3 \mathrm{~s}$. The fault location is shown in Fig. 5. Figure 6a presents the dynamic curves of the generator rotor angle

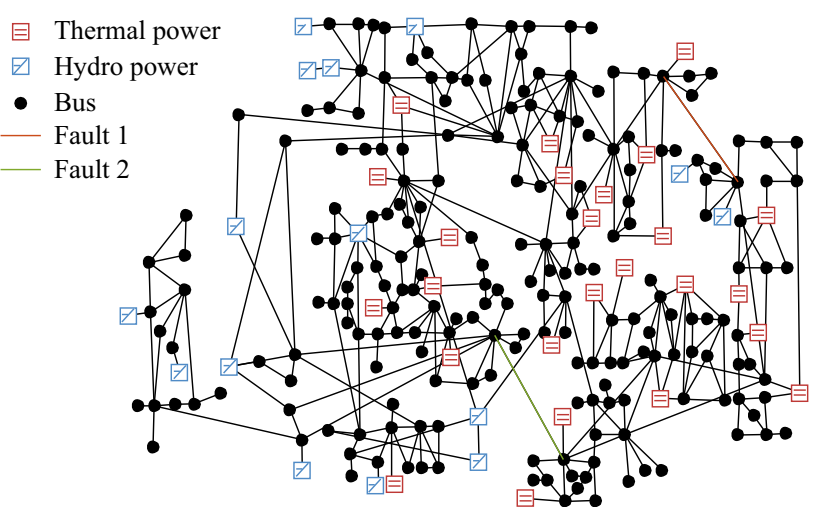

Fig. 5 RPPS topology, generator distribution and fault position 


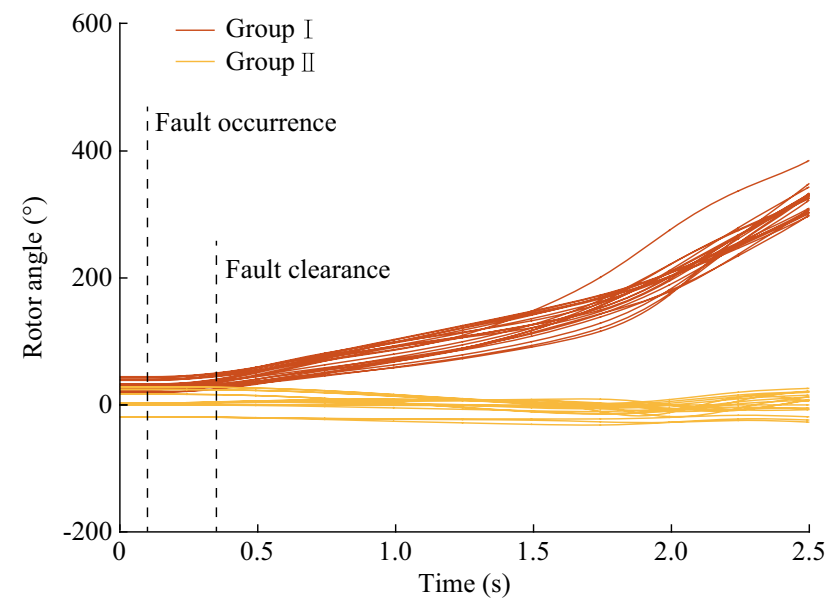

(a) Rotor angles of pre-split generators

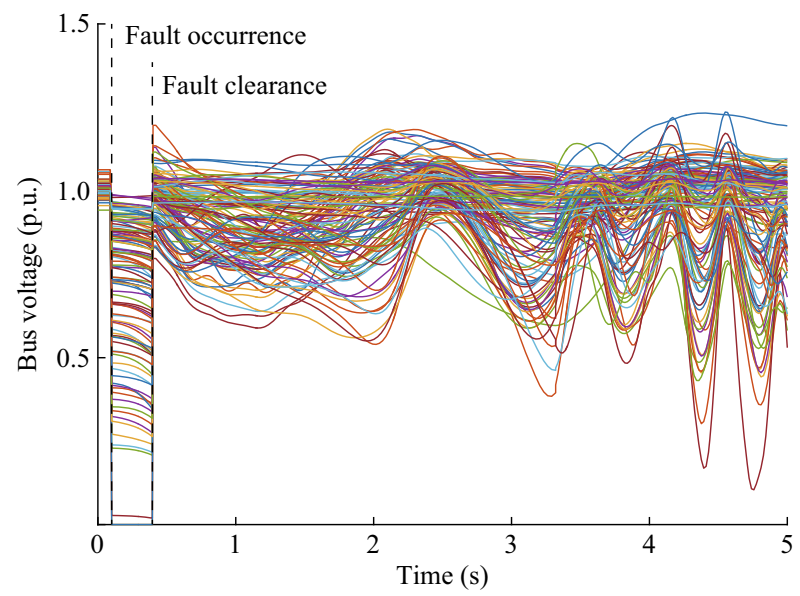

(b) Voltages of pre-split buses

Fig. 6 System dynamic without splitting control after fault 1

after fault 1. An out-of-step oscillation between the two generator groups is clearly induced by the fault. Accordingly, RPPS is supposed to be split into two islands to separate these two asynchronous generator groups.

The optimal splitting strategies presented in Fig. 7a. Suppose that the splitting strategy is activated $2 \mathrm{~s}$ after the fault clearance, including the SSS computation time of approximately $1.2 \mathrm{~s}$ and the SSS preparation work and data transfer (assumed to be $0.8 \mathrm{~s}$ in total). With 14 transmission lines tripped out, the asynchronous generator groups are successfully divided into two isolated islands with feasible power flow solutions.

Furthermore, Fig. 7 investigates the island stability performance. Without the splitting control, the out-of-step oscillation between the two generator groups induces severe angle and voltage instability, and finally leads to the system-wide collapse, as shown in Fig. 6. In contrast, by executing splitting control with the properly-selected strategy shown in Fig. 7a, the inter-area oscillation is eliminated and each island can separately damp the

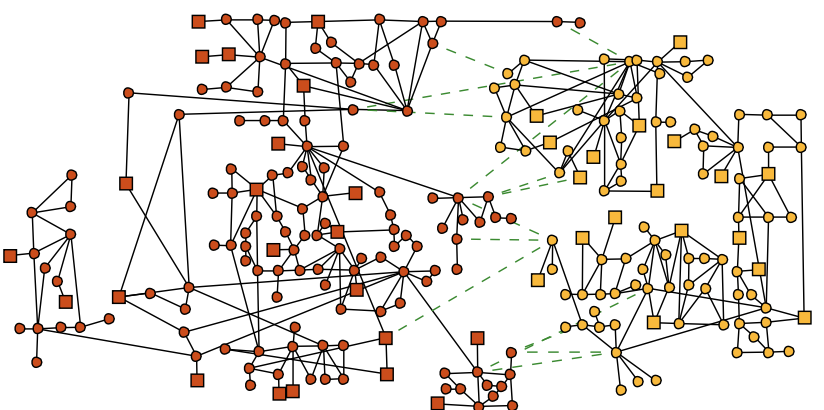

$\square$ Generator in island I ; $\square$ Generator in island II

○ Bus in island I ; o Bus in island II ; . - Tripped-out branch

(a) Optimal splitting strategy

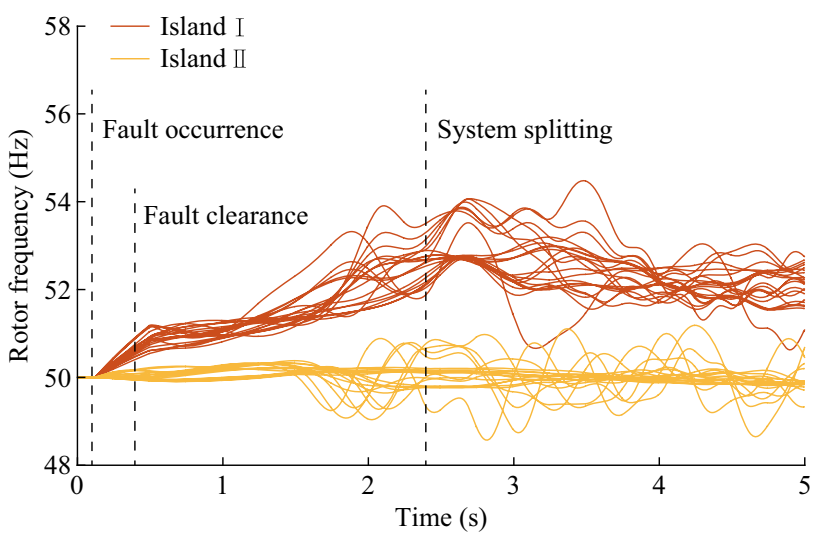

(b) Rotor speeds of post-split generators

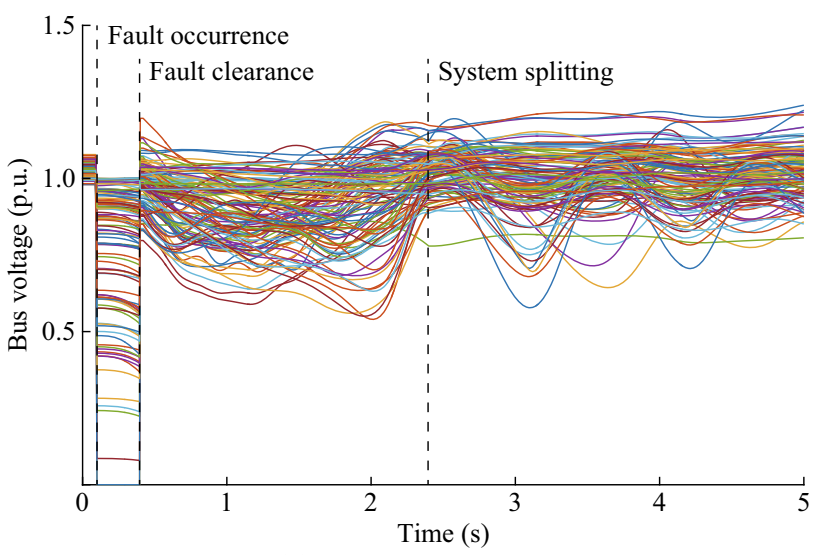

(c) Voltages of post-split buses

Fig. 7 Splitting strategy for fault 1 and island stability performance: a two-group oscillation case

generator oscillation as presented in Fig. 7b. Comparing Figs. $7 \mathrm{c}$ and $6 \mathrm{~b}$, it can be observed that the potential voltage collapse risk induced by the out-of-step oscillation is also effectively refrained on each island. The transient stability performance verifies the feasibility of the adopted splitting strategy.

Additionally, Table 1 summarizes the load shedding price of the obtained splitting strategy. In Table $1, \sum P^{G}$, $\sum P^{L}$ separately represent the power generation and power 
Table 1 Island power balance and power load shedding conditions of optimized splitting strategy for fault 1

\begin{tabular}{llllll}
\hline Condition & Island & $\sum P^{G}$ (p.u.) & $\sum P^{L}$ (p.u.) & $\Delta P^{\text {per }}$ (p.u.) & $\Delta P^{\text {tem }}$ (p.u.) \\
\hline Before splitting & Island I & 87.13 & 79.90 & - & - \\
& Island II & 46.13 & 53.24 & - & 0 \\
After splitting & Island I & 79.90 & 79.90 & 0 & 0 \\
& Island II & 53.24 & 53.24 & 0 \\
\hline
\end{tabular}

load amount within each island; $\Delta P^{p e r}$ represents the steady-state load shedding, relating to $O_{\text {basic }}$ in (23); $\Delta P^{\text {tem }}$ represents the transient load shedding, relating to $O_{T E C}$ in (23). It can be seen that before the disturbance, the power flow direction on the tripped transmission lines is from island I to island II. After the splitting control, each island holds power balance and maintains power supply separately by generation regulation and re-dispatch. However, in the transient process on island II, the frequency dip risk leads to some temporary load losses. This phenomenon is induced by the inadequate frequency response ability of island II. As a power-receiving region, island II originally holds a $15 \%$ imbalance between load and generation before splitting. Thus, after the system splitting, the corresponding generators should immediately increase their generation to prevent the unacceptable frequency dip. However, the generators in island II are primarily conventional thermal power plants, whose regulation speeds are relatively slow. Consequently, $5.66 \%$ power load is shed in the transient process to maintain the frequency dynamics, which can be recovered after the island reaches a steady state.

\subsubsection{A multi-group oscillation case}

Splitting control for fault 2 represents a typical multigroup oscillation case. The fault location is shown in Fig. 8a. With a three-phase short circuit fault lasting for 0.3 $\mathrm{s}$, the generator rotor angle dynamic (as shown in Fig. 8a) indicates an inter-area oscillation among three groups of generators. Hence, the system should be split into three isolated power islands. The optimized splitting strategy is presented in Fig. 9, which effectively separates the three asynchronous generator groups. Figure $8 \mathrm{~b}$, c together illustrate the transient feasibility of the obtained splitting strategy. This case indicates the practicability of the proposed approach in the multi-group oscillation case.

Additionally, the performance indices of the optimized splitting strategy is summarized in Table 2, including the island power balance situation, the power load shedding amount and the VSM. It is obvious that island III is the receiving region with over $50 \%$ power load services from other regions in the pre-split steady state. Thus, the limited generation regulation capacity on island III causes $37.18 \%$

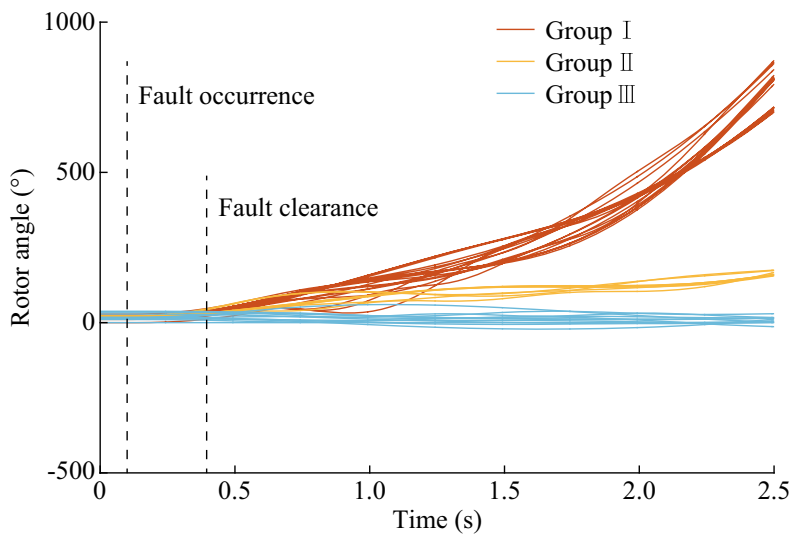

(a) Rotor angles of pre-split generators

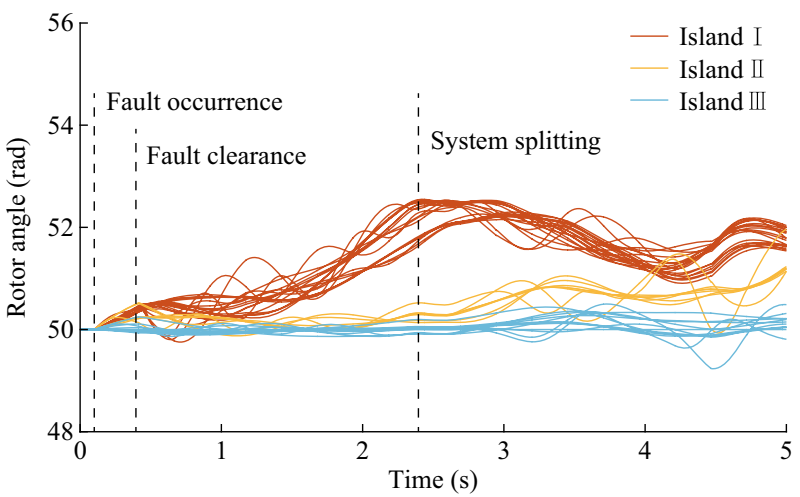

(b) Rotor speeds of post-split generators

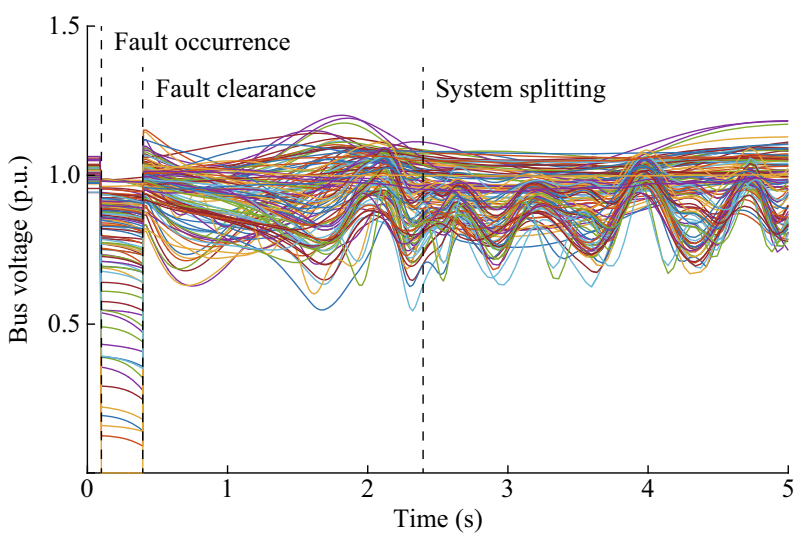

(c) Voltages of post-split buses

Fig. 8 Splitting strategy for fault 2 and island stability performance: a multi-group oscillation case 


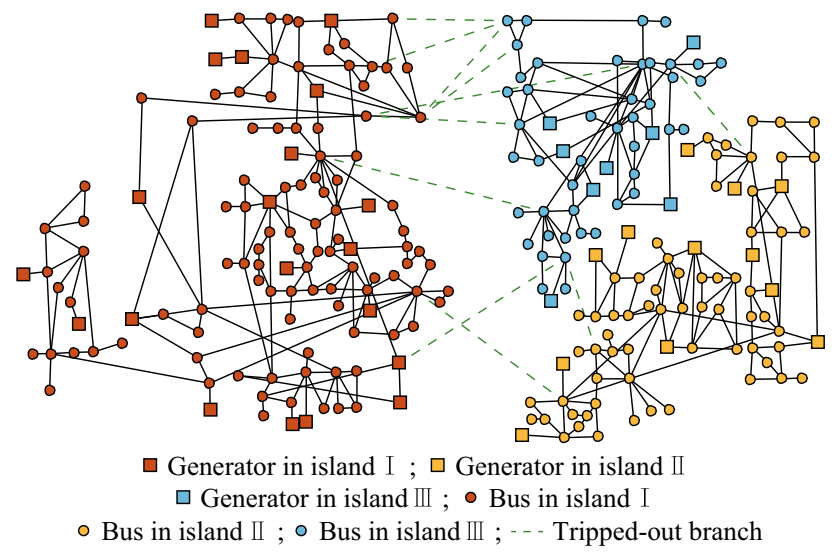

Fig. 9 Optimal splitting strategy for fault 2

power load shedding considering the transient frequency dip.

\subsection{Method necessity analysis}

In the following, the splitting case under fault 1 is taken as a representative to further discuss the effect and necessity of considering SEC and TEC for improving the island static and transient performance. A comparison of splitting strategies with and without stability considerations is presented. Denote the splitting strategy obtained by the proposed SSS approach, namely the strategy shown in Fig. 7a, as S0. The comparative strategies are generated by the SSS model proposed in [23], which aims to minimize steadystate load shedding with the constraints of synchronization separation and DCPF only. This model is denoted as M1. As demonstrated in [23], there exist MOSs for M1. And using the conventional optimization algorithms can only obtain one optimal solution randomly. This fact indicates that the strategies generated by M1 are not unique in every computation and do not provide the island stability information for the system operators. Although specific algorithms can be used to obtain all the optimal solutions for M1, the stability performance of all the candidate strategies should be further checked and compared, which introduces extra computational time.

Two splitting strategies are stochastically generated by M1 and they are denoted as $\mathrm{C} 1$ and $\mathrm{C} 2$. Table 3 presents the comparison between $\mathrm{S} 0, \mathrm{C} 1$ and $\mathrm{C} 2$. First, note that all three of these strategies achieve complete power supply (i.e., no steady-state power load loss) while satisfying conventional DCPF and SSC. This phenomenon is consistent with the MOS feature of M1 revealed in [23]. However, although $\mathrm{C} 1$ and $\mathrm{C} 2$ are capable of separating asynchronous generator groups and maintaining the DCPF constraint, they may not be the best choice, or even a feasible choice, for practical operation considering the island stability issues.

1) Steady-state performance comparison. More specifically, for the steady-state stability performance, Table 3 illustrates that without the consideration of reactive power flow, the power islands formed in $\mathrm{C} 1$ are actually incapable of obtaining a feasible AC power flow operating point. Although the conventional DCPF result shows no abnormality, indicating that the active power supply is adequate, $\mathrm{C} 1$ is unreasonable for voltage support and reactive power distribution. These types of strategies are clearly unacceptable in practical operation and should be rejected in the SSS process. In contrast to $\mathrm{C} 1, \mathrm{C} 2$ and S0 both possess feasible operating points on each island. However, C2 is inferior to S0 considering the island stability margin performance. This result indicates that $\mathrm{C} 2$ is a worse choice compared with S0 from the perspective of static stability margin despite its feasibility in power flow solving.

2) Transient performance comparison. For the transient stability performance, a frequency dip risk exists on island II due to its restricted frequency response capacity, as discussed previously. Although no power load shedding is induced in the steady state considering generation capacity, the frequency dynamics leads to a considerable temporary load loss. Specifically, C1 and C2 respectively induce $26.29 \%$ and $13.15 \%$ load sheddings in the transient process to prevent the frequency dip. This power load loss is largely avoidable, but it is not sufficiently recognized due

Table 2 Splitting performance of optimized strategy for fault 2

\begin{tabular}{|c|c|c|c|c|c|c|}
\hline Condition & Island & $\sum P^{G}$ (p.u.) & $\sum P^{L}$ (p.u.) & $\Delta P^{p e r}$ (p.u.) & $\Delta P^{t e m}$ (p.u.) & VSM \\
\hline \multirow[t]{3}{*}{ Before splitting } & Island I & 79.31 & 56.89 & - & - & - \\
\hline & Island II & 26.15 & 19.61 & - & - & - \\
\hline & Island III & 27.67 & 56.65 & - & - & \\
\hline \multirow[t]{3}{*}{ After splitting } & Island I & 56.89 & 56.89 & 0 & 0 & 0.0842 \\
\hline & Island II & 19.61 & 19.61 & 0 & 0 & 0.0298 \\
\hline & Island III & 56.65 & 56.65 & 0 & 21.06 & 0.0592 \\
\hline
\end{tabular}


Table 3 Performance comparison of different splitting strategies

\begin{tabular}{|c|c|c|c|c|c|c|}
\hline \multirow[t]{2}{*}{ Strategy } & \multirow[t]{2}{*}{ Tripped branch number } & \multirow[t]{2}{*}{ Power flow feasible? } & \multicolumn{2}{|c|}{ VSM (L-index) } & \multicolumn{2}{|c|}{$\Delta P^{t e m}$ (р.u.) } \\
\hline & & & Island I & Island II & Island I & Island II \\
\hline So & 14 & Yes & 0.2222 & 0.0599 & 0 & 3.01 \\
\hline $\mathrm{C} 1$ & 10 & No & - & - & 0 & 14.00 \\
\hline $\mathrm{C} 2$ & 13 & Yes & 0.3172 & 0.0600 & 0 & 7.00 \\
\hline
\end{tabular}

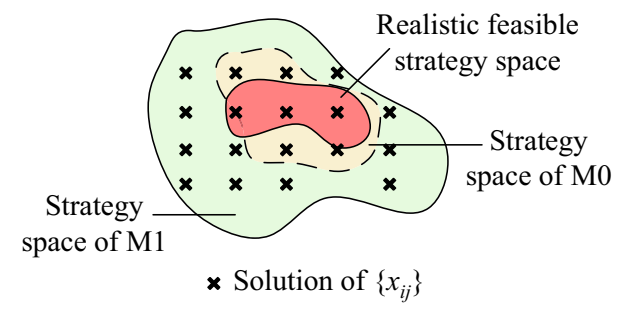

Fig. 10 Illustration of strategy spaces of M0 and M1 defined in space $\left\{x_{i j} \mid \forall(i, j) \in E\right\}$

to neglecting TEC in model M1. In contrast, S0 only causes $5.66 \%$ load shedding. This result indicates the necessity of considering TEC.

Theoretically, M0 presents superiority over M1 because it depicts a modified substitution of the realistic splitting strategy space, as illustrated in Fig. 10. Clearly, it is difficult to directly depict the realistic splitting strategy space considering the precise model of all the transient and static processes during the system islanding. Rather, both M0 and M1 attempt to investigate a simplification or an approximation of the realistic strategy space. In the approximated strategy space, SSS can be performed more easily. However, the complete neglect of stability considerations leads M1 to an over-optimistic extension of the realistic feasible strategy space. Thus, the strategies obtained from M1 have a high potential of locating beyond the realistic strategy space, as illustrated by the green area in Fig. 10. These strategies (such as $\mathrm{C} 1$ ) result in unsatisfactory stability performance. Although some solutions may be obtained within the feasible strategy space, such as $\mathrm{C} 2$, it is rather stochastic. This uncontrollable and stochastic decision process would be unacceptable in practical utilization. In contrast, M0 provides a more cautious substitution of the realistic strategy space. With an appropriate formulation of both the static and transient stability characteristics, the irrational strategies with poor stability performance can be largely rejected. Thus, a splitting strategy with better static and transient equilibriums is selected to ensure island stability performance.
Table 4 Strategy comparison with different emphases on SEC and TEC

\begin{tabular}{llllll}
\hline Objective weights in (23) & \multicolumn{2}{l}{ VSM } & & & \multicolumn{2}{l}{$\Delta P^{\text {tem }}$ (p.u.) } \\
\cline { 2 - 3 } & Island I & Island II & & Island I & Island II \\
\hline$\left(\lambda_{1}, \lambda_{2}, \lambda_{3}\right)$ & 0.2222 & 0.0599 & & 0 & 3.01 \\
$\left(\lambda_{1}, 0, \lambda_{3}\right)$ & 0.3537 & 0.0600 & & 0 & 2.12 \\
$\left(\lambda_{1}, \lambda_{2}, 0\right)$ & 0.1924 & 0.0601 & & 0 & 8.92 \\
$\left(\lambda_{1}, 0,0\right)$ & 0.3332 & 0.0603 & & 0 & 10.74 \\
\hline
\end{tabular}

\subsection{Further discussion}

As presented in Section 3.4, M0 is a multi-objective optimization model. Thus, the trade-off between less load shedding and higher stability margin can be adjusted by the objective weights $\lambda_{1}, \lambda_{2}, \lambda_{3}$ in (23). Table 4 tabulates several strategies with different emphases on SEC and TEC. Since minimizing the steady-state load shedding is of the highest priority, $\lambda_{1}$ is fixed. Setting $\lambda_{2}$ to 0 indicates a higher priority of transient load supply requirement over steady-state stability. Consequently, only $3.63 \%$ transient load shedding is induced, although it breaks other stability considerations to some extent. Investigating the island topologies under different objective weights, it can be found that the buses of low VSM are concentrated in areas with chain structures, and without the VSM consideration, this impractical network structure is not detected. Setting $\lambda_{3}$ to 0 leads to the splitting strategy with a higher VSM on island I but more load shedding in the transient process. Specifically, $19.75 \%$ load shedding is induced to prevent the frequency dip in this strategy. When setting $\lambda_{2}$ and $\lambda_{3}$ to 0 , the model degrades into SSS without any stability considerations and results in a stochastic performance on island stability characteristics.

Further, to investigate the adaptability of the proposed approach, Table 5 provide simulation results for more stochastical faults that may reduce inter-area oscillation. In each case, the splitting strategy effectively divides the asynchronous generators into isolated islands of a certain number, which is decided by the number of coherency generator groups. The feasible steady-state operation point and the island oscillation damping are both guaranteed, 
Table 5 Method performance test for stochastic cases

\begin{tabular}{llllll}
\hline Fault case & Island number & Island VSM & Steady-state load shedding $(\%)$ & Transient load shedding $(\%)$ & Computational time $(\mathrm{s})$ \\
\hline Fault 1 & 2 & $(0.2222,0.0599)$ & $(0,0)$ & $(0,5.66)$ & 1.0492 \\
Fault 2 & 3 & $(0.0842,0.0298,0.0592)$ & $(0,0,0)$ & $(0,0,37.18)$ & 0.6988 \\
Fault 3 & 2 & $(0.2085,0.0820)$ & $(0,0)$ & $(0,19.18)$ & 0.9490 \\
Fault 4 & 3 & $(0.2076,0.0794,0.0385)$ & $(0,0,0)$ & $(0,12.28,0)$ & 0.7213 \\
Fault 5 & 2 & $(0.2663,0.0638)$ & $(0,0)$ & $(0,6.64)$ & 0.8879 \\
Fault 6 & 2 & $(0.2407,0.0757)$ & $(0,0)$ & $(0,10.03)$ & 1.0281 \\
\hline
\end{tabular}

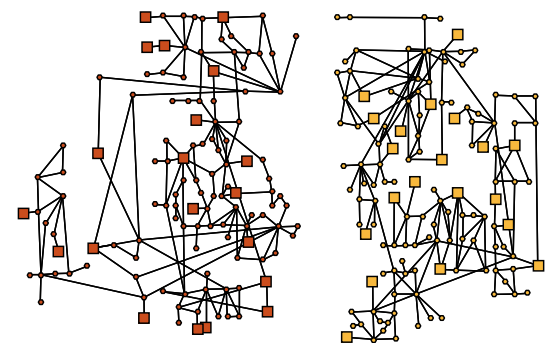

$\square$ Generator in island I; ○ Bus in island I $\square$ Generator in island II; o Bus in island II

(a) Fault 3: splitting strategy

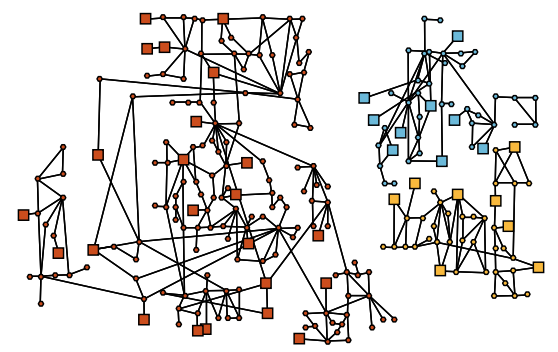

$\square$ Generator in island I ; ○ Bus in island I $\square$ Generator in island $I I$; $\circ$ Bus in island II $\square$ Generator in island III; o Bus in island III (d) Fault 4: splitting strategy

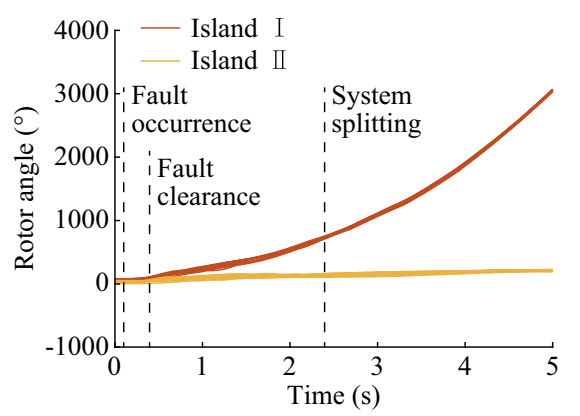

(b) Fault 3: rotor angles of post-split generators

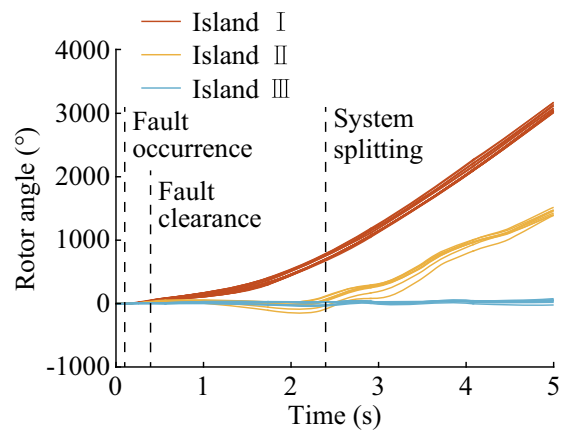

(e) Fault 4: rotor angles of post-split generators

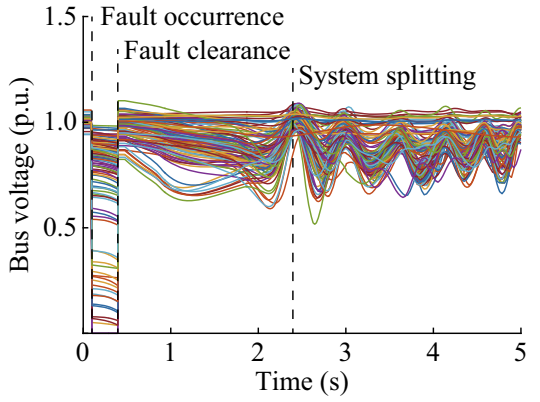

(c) Fault 3: voltages of post-split buses

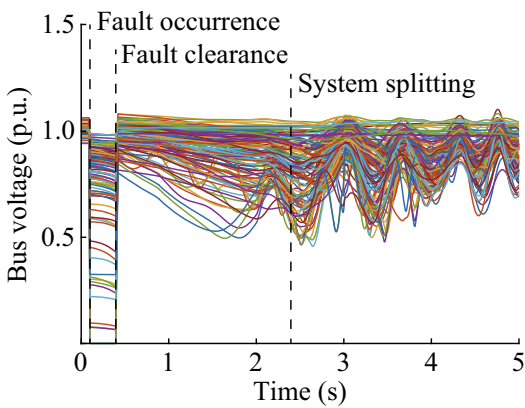

(f) Fault 4: voltages of post-split buses

Fig. 11 Splitting strategy and island stability performance for stochastic cases

with an optimal load shedding amount in the transient process and the steady state. Figure 11 presents the optimal splitting strategy and the corresponding post-split transient performance for some of the cases. As the figures illustrate, the severe instability threat induced by the inter-area oscillation is effectively refrained by forming separatestable islands. Although each island can not directly reach the steady state by only executing splitting control, the system splitting ensures the basic island stability and restrains the risk of system-wide collapse, which is the most important target of splitting control. In the subsequent operation, each island can adopt proper assistant control methods to separately damp the oscillation, which is much slighter and easier to handle compared with the original severe inter-area oscillation.

As for the computational efficiency, adding SEC and TEC into the SSS model indeed increases the computation time, but it is still acceptable for real-time control. The simulation results provided in Table 5 verifies the method practicability. It can be seen that in different oscillation cases, the proposed SSS model can be effectively solved within about one second, which is usable for the splitting control execution. The solving efficiency benefits from both the superior optimization properties of the MISOCP problems and the splitting strategy space reduction technique [17]. For highly complicated and large-scale power systems, we recommend performing off-line or on-line 
power network simplification before the real-time splitting strategy decision to accelerate the searching process.

\section{Conclusion}

This paper proposes a MISOCP-based SSS approach with consideration of island stability. The static stability and transient stability issues in splitting control are investigated as well as their mathematical formulation in SSS. By embedding the island stability issues in SSS, the proposed method can provide a more practical splitting strategy and make a comprehensive trade-off between island stability and load shedding amount.

The consideration of SEC and TEC is vital to enhance island static and transient stability during and after the splitting control. The proposed method has two advantages over previous works. First, it addresses the essential stability issues in both the steady state and the transient process. These considerations can effectively directly reject the splitting strategies with poor stability performance. Second, the SSS problem is formulated as a deterministic MISOCP problem. Compared with the previous heuristic search methods or the non-linear optimization methods, this MISOCP-based method exhibits satisfactory problemsolving efficiency and is able to converge to a deterministic and optimal solution rather than a stochastic solution.

In this work, we simply adopt a general-purpose solver, i.e., CPLEX, for model optimization. A new optimization algorithm is a topic for future work to further enhance the computational efficiency. Besides, this paper adopts a linearized model for the island transient frequency process, as a trade-off between the handling the stability issues and the ensuring the real-time decision. This is based on a rational assumption that the spitting control is activated timely so that the severest island instability threat is the frequency dip/rise caused by the certain power imbalance. However, if the execution of splitting control is largely delayed, the unbalanced active power on the island might further induce severe voltage stability issue. And in this case the ignorance of the voltage dynamic becomes impractical. It is another topic that is on-going to deal with the transient voltage stability issue.

Open Access This article is distributed under the terms of the Creative Commons Attribution 4.0 International License (http:// creativecommons.org/licenses/by/4.0/), which permits unrestricted use, distribution, and reproduction in any medium, provided you give appropriate credit to the original author(s) and the source, provide a link to the Creative Commons license, and indicate if changes were made.

\section{References}

[1] Xue YS (2006) Space-time cooperative framework for defending blackouts: part III optimization and coordination of defense lines. Autom Electr Power Syst 30(3):1-10

[2] Liu Y, Liu Y (2006) Aspects on power system islanding for preventing widespread blackout. In: Proceedings of 2006 IEEE international conference on networking, sensing and control, Lauderdale, USA, 23-25 April 2006, pp 1090-1095

[3] Sun K, Zheng D, Lu Q (2003) Splitting strategies for islanding operation of large-scale power systems using OBDD-based methods. IEEE Trans Power Syst 18(2):912-923

[4] Kamali S, Amraee T, Bathaee SMT (2016) Prediction of unplanned islanding using an energy based strategy. IET Gener Transm Distrib 10(1):183-191

[5] Salimian MR, Aghamohammadi MR (2016) Intelligent out of step predictor for inter area oscillations using speed-acceleration criterion as a time matching for controlled islanding. IEEE Trans Smart Grid 9(4):2488-2497

[6] Sun K, Zhao Q, Zheng DZ et al (2002) A two-phase method based on OBDD for searching for splitting strategies of largescale power systems. In: Proceedings of international conference on power system technology, Kunming, China, 13-17 October 2002, pp 834-838

[7] Shen C, Wu JY, Qiao Y et al (2006) Studies on active splitting control of power systems. Proc CSEE 26(13):1-6

[8] You H, Vittal V, Wang X (2004) Slow coherency-based islanding. IEEE Trans Power Syst 19(1):483-491

[9] Yang B, Vittal V, Heydt GT et al (2007) A novel slow coherency based graph theoretic islanding strategy. In: Proceedings of IEEE PES general meeting, Tampa, USA, 24-28 June 2007, pp 1-7

[10] Wang X, Vittal V (2004) System islanding using minimal cut sets with minimum net flow. In: Proceedings of IEEE PES power systems conference and exposition, New York, USA, 10-13 October 2004, pp 379-384

[11] Ding L, Gonzalez-Longatt FM, Wall P et al (2013) Two-step spectral clustering controlled islanding algorithm. IEEE Trans Power Syst 28(1):75-84

[12] Trodden PA, Bukhsh WA, Grothey A et al (2012) MILP islanding of power networks by bus splitting. In: Proceedings of IEEE PES general meeting, San Diego, USA, 22-26 July 2012, pp 1-8

[13] El-Werfelli M, Brooks J, Dunn R (2008) Controlled islanding scheme for power systems. In: Proceedings of 18th international conference on intelligent system application to power systems, Porto, Portugal, 11-16 September 2015, pp 1-6

[14] Ding T, Sun H, Sun K et al (2015) Graph theory based splitting strategies for power system islanding operation. In: Proceedings of IEEE PES general meeting, Denver, USA, 26-30 July 2015, pp 1-6

[15] Golari M, Fan N, Wang J (2016) Large-scale stochastic power grid islanding operations by line switching and controlled load shedding. Energy Syst 8(3):1-21

[16] Jabari F, Seyedi H, Ravadanegh SN (2015) Large-scale power system controlled islanding based on backward elimination method and primary maximum expansion areas considering static voltage stability. Int $\mathrm{J}$ Electr Power Energy Syst 67:368-380

[17] Zhou Y, Hu W, Min Y et al (2016) MILP-based splitting strategy searching considering island connectivity and voltage stability margin. In: Proceedings of IEEE PES general meeting, Boston, USA, 17-21 July 2016, pp 1-6

[18] Sun K, Zheng DZ, Lu Q et al (2005) A simulation study of OBDD-based proper splitting strategies for power systems 
under consideration of transient stability. IEEE Trans Power Syst 20(1):389-399

[19] Song H, Wu J, Wu K (2014) A wide-area measurement systemsbased adaptive strategy for controlled islanding in bulk power systems. Energies 7(4):2631-2657

[20] Koohi-Kamali S, Rahim NA (2016) Coordinated control of smart microgrid during and after islanding operation to prevent under frequency load shedding using energy storage system. Energy Convers Manag 127:623-646

[21] Ding L, Ma Z, Wall P et al (2017) Graph spectra based controlled islanding for low inertia power systems. IEEE Trans Power Deliv 32(1):302-309

[22] Quirs-Torts J, Demetriou P, Panteli M et al (2017) Intentional controlled islanding and risk assessment: a unified framework. IEEE Syst J 12(4):3637-3648

[23] Ding T, Sun K, Huang C et al (2015) Mixed-integer linear programming-based splitting strategies for power system islanding operation considering network connectivity. IEEE Syst J 12(1):350-359

[24] Adams WP, Sherali HD (1990) Linearization strategies for a class of zero-one mixed integer programming problems. Oper Res 38(2):217-226

[25] Wang Z, Hu X (2008) Analysis of static angularity and voltage stability criteria. Electr Power Autom Equip 28(2):61-64

[26] Kessel P, Glavitsch H (1986) Estimating the voltage stability of a power system. IEEE Trans Power Deliv 1(3):346-354

[27] Amraee T, Saberi H (2017) Controlled islanding using transmission switching and load shedding for enhancing power grid resilience. Int J Electr Power Energy Syst 91:135-143

[28] Kundur P (1994) Power system stability and control. McGrawHill, New York

[29] Chavez H, Baldick R, Sharma S (2014) Governor rate-constrained OPF for primary frequency control adequacy. IEEE Trans Power Syst 29(3):1473-1480

[30] Rudez U, Mihalic R (2016) WAMS-based underfrequency load shedding with short-term frequency prediction. IEEE Trans Power Deliv 31(4):1912-1920

[31] Alizadeh F, Goldfarb D (2003) Second-order cone programming. Math Program 95(1):3-51
[32] Anaparthi KK, Chaudhuri B, Thornhill NF et al (2005) Coherency identification in power systems through principal component analysis. IEEE Trans Power Syst 20(3):1658-1660

[33] Gacic N, Zecevic AI, Siljak DD (1998) Coherency recognition using epsilon decomposition. IEEE Trans Power Syst 13(2):314-319

[34] Isazadeh G, Khodabakhshian A, Gholipour E (2015) New intelligent controlled islanding scheme in large interconnected power systems. IET Gener Transm Distrib 9(16):2686-2696

Yifan ZHOU received the B.S. degree in electrical engineering from Tsinghua University, Beijing, China, in 2014, where she is currently pursuing the Ph.D. degree. Her research interests include modelling and optimal scheduling of the integrated power and heat system.

Wei HU received the B.S. and Ph.D. degrees in electrical engineering from Tsinghua University, Beijing, China, in 1998 and 2002, respectively. Currently, he is working as an associate professor with the Department of Electrical Engineering, Tsinghua University. His research interests include integrated energy system operation, deep learning in power system modeling and security analysis, and smart control.

Yong MIN received the B.S. degree and the Ph.D. degree in electrical engineering from Tsinghua University, Beijing, China, in 1984 and 1990 , respectively. Currently, he is working as a professor with the Department of Electrical Engineering, Tsinghua University. His research interests include integrated energy system operation, power system stability and control.

Le ZHENG received the B.S. and Ph.D. degrees in electrical engineering from Tsinghua University, Beijing, China, in 2011 and 2017, respectively. Currently, he is a postdoc in Stanford University. His research interests include power system security analysis and control, and deep learning technique in power system analysis. 